\title{
Tratamiento conservador de la artrosis de cadera
}

\author{
Conservative treatment of hip osteoarthritis
}

Maria Celia Iñurrateguia (iD)

\begin{abstract}
Resumen
Ante un escenario clínico de coxalgia por artrosis de cadera se planteó la necesidad de conocer los tratamientos conservadores más seguros y efectivos para el manejo del dolor. El tratamiento de la artrosis requiere un enfoque integral e individualizado en función de las preferencias del paciente para lograr el máximo beneficio clínico. Existen numerosas estrategias útiles para el manejo del dolor en pacientes con artrosis de cadera siendo fuertemente recomendados de inicio la actividad física, los antiinflamatorios no esteroideos (AINE) orales y en ciertos casos los corticoides intraarticulares, tramadol o duloxetina, siempre asociado con la actividad física. Los ejercicios más recomendados son los aeróbicos y el Tai Chi o yoga.

\section{Abstract}

Faced with a clinical scenario of coxalgia due to hip osteoarthritis, the need to know the safest and most effective conservative treatments for pain management arose. The treatment of osteoarthritis requires a comprehensive and individualised approach based on the patient's preferences to achieve maximum clinical benefit. There are numerous useful strategies for pain management in patients with hip osteoarthritis being strongly recommended from the beginning such as physical activity, oral non-steroidal anti-inflammatory drugs (NSAID) and in certain cases intra-articular corticosteroids, tramadol or duloxetine, always associated with physical activity. The most recommended exercises are aerobics and Tai Chi or yoga.
\end{abstract}

Palabras clave: artrosis de cadera, tratamiento conservador. Keywords: hip osteoarthritis, conservative treatment.

Iñurrategui MC. Tratamiento conservador de la artrosis de cadera. Evid Actual Pract Ambul. 2021;24(2):e002071. Available from: https://dx.doi.org/10. 51987/evidencia.v24i2.6937.

\section{Escenario clínico}

Acude al consultorio de demanda espontánea una paciente de 80 años que consulta por coxalgia derecha de varios meses de evolución. Refiere que el dolor fue de inicio insidioso y que al principio predominaba al despertarse, momento en el que se asociaba también a rigidez. Desde ese entonces comenta también episodios de bloqueo en la articulación al caminar y en ocasiones, dolor durante la noche. Niega haber sufrido algún traumatismo y no está tomando actualmente medicaciones para aliviar el dolor.

Al examen físico se encuentra limitación a la movilidad pasiva de la cadera derecha por lo que se solicitan radiografías en las que se observan signos de artrosis de ambas caderas con predominio derecho.

\section{Pregunta que genero el caso}

En pacientes con coxalgia debida a artrosis de cadera, ¿Cuáles son los tratamientos conservadores (farmacológicos y no farmacológicos) más seguros y efectivos para el manejo del dolor?

\section{Estrategia de búsqueda}

Se realizó una búsqueda bibliográfica en PubMed utilizando los términos (hip osteoarthritis[MeSH Terms]) AND 'pain management' y en Trip Database con los términos (chronic pain due to hip osteoarthritis) AND (pain management). En ambas bases se limitaron los resultados a ensayos clínicos, revisiones sistemáticas, metaanálisis y guías de práctica clínica publicados durante los últimos cinco años.

De los resultados obtenidos en las diferentes búsquedas se eligieron aquellos artículos y guías de práctica clínica más recientes y con mayor calidad de evidencia.

\section{Algunos aspectos acerca de la artrosis de cadera}

La artrosis, u osteoartritis, es una enfermedad crónica que puede afectar cualquier articulación sinovial; se trata de un proceso dinámico de destrucción y reparación ${ }^{1}$. La degeneración que lleva a la pérdida progresiva del cartílago articular se acompaña de la remodelación ósea con formación ósea reactiva y crecimiento de osteofitos.

El compromiso de grandes articulaciones de los miembros inferiores, incluidas las caderas, puede ocasionar una alta morbilidad y discapacidad física, teniendo como consecuencia el aumento en el uso del sistema de salud. En un estudio realizado en los EE.UU. se observó que el $27 \%$ de los adultos mayores a 45 años presentaban signos radiológicos de artrosis de cadera, pero solo un $9,2 \%$ tenían síntomas asociados a ésta ${ }^{2}$.

El diagnóstico se realiza principalmente a través del interrogatorio y el examen físico, y pueden utilizarse estudios por imágenes para su confirmación y seguimiento.

Los síntomas más frecuentes son rigidez y dolor articular a predominio matutino o luego de estar en reposo, y dolor que también empeora luego de realizar actividad vigorosa. Habitualmente el dolor se presenta a nivel de la ingle o el muslo e irradia a los glúteos o la rodilla. El paciente puede manifestar la sensación de bloqueo o crepitación a la movilización, y con el tiempo puede llevar a limitación de la movilidad articular, afectando la habilidad de caminar.

En el examen físico se debe evaluar visualmente la simetría entre ambos miembros inferiores, palpar la articulación afectada evaluando la presencia de tumoraciones o dolor localizado, y comparar la movilidad de ambas caderas.

\section{Resumen de la evidencia}

Se seleccionaron los tres artículos más relevantes para responder a la pregunta planteadas: una guía de práctica clínica basada en la evidencia ${ }^{3}$, una revisión sistemática de revisiones sistemáticas ${ }^{4}$ y un meta-análisis en red ${ }^{5}$.

\footnotetext{
a Servicio de Medicina Familiar y Comunitaria, Hospital Italiano de Buenos Aires. maria.inurrategui@hospitalitaliano.org.ar
} 
Kolasinski SL, Neogi T, Hochberg MC, et al. 2019 American College of Rheumatology/Arthritis Foundation Guideline for the Management of Osteoarthritis of the Hand, Hip, and Knee. Arthritis Care Res (Hoboken) 2020;72(2):149-162.

Esta guía de práctica clínica basada en la evidencia ${ }^{3}$ actualizó las recomendaciones del Colegio Americano de Reumatología para el manejo de artrosis de mano, cadera y rodilla.

Se realizó una revisión sistemática de la literatura para resumir la evidencia acerca de los beneficios y daños de las diferentes estrategias terapéuticas utilizadas para el tratamiento de la artrosis. Se utilizó la metodología GRADE para evaluar la certeza de la evidencia y se conformó un panel de expertos (integrado por médicos reumatólogos, médicos internistas, terapistas ocupacionales, fisioterapeutas y pacientes) para arribar de manera consensuada a las recomendaciones (ver Tabla 1).

\section{Conclusiones del estudio}

En cuanto a las medidas no farmacológicas la realización de actividad física está fuertemente recomendada para el manejo del paciente con artrosis de cadera pero no hay evidencia suficiente para prescribir un tipo específico de ejercicio. La indicación de actividad física debe considerar las preferencias y posibilidades del paciente, así como las barreras para concretarlo. La mayoría de los estudios evaluaron caminata, ciclismo supervisado en bicicletas fijas, ejercicios de fortalecimiento y ejercicio acuático. El beneficio del Tai Chi en la recuperación de fuerza muscular, balance corporal y prevención de caídas, así como en la mejoras de depresión y autonomía podrían reflejar el impacto holístico de esta práctica que integra mente y cuerpo. El descenso de peso es otra de las recomendaciones fuertes, ya que se observó que una pérdida de más del $5 \%$ del índice de masa corporal en pacientes con sobrepeso u obesidad puede generar cambios en resultados clínicos y mecánicos, lo cual se potencia con la asociación de programas de actividad física. El uso del bastón está fuertemente recomendado como dispositivo de asistencia en aquellos pacientes en los que la enfermedad está causando dolor intenso con gran impacto en la deambulación y la estabilidad de la articulación.

Entre las medidas farmacológicas fuertemente recomendadas, los antiinflamatorios no esteroideos (AINE) orales continúan siendo el primer escalón del tratamiento farmacológico para la artrosis de cadera, siempre y cuando se utilicen por un intervalo corto de tiempo y teniendo en cuenta sus potenciales efectos adversos.

El tamaño del efecto del paracetamol es pequeño y la monoterapia puede ser inefectiva. Se recomiendan cursos cortos y episódicos en aquellos casos donde otras medidas farmacológicas están contraindicadas. La duloxetina es eficaz como monoterapia o en combinación con AINE, pero se deben tener en cuenta la tolerancia y los efectos adversos al momento de indicarla. En cuanto al tramadol, nuevos estudios muestran beneficios modestos en el tratamiento a mediano plazo (de tres meses a un año) y se recomienda principalmente en aquellos pacientes con contraindicación a los AINE y/o que no sean candidatos a realizar un tratamiento quirúrgico.

Tabla 1. Tipo de recomendaciones para el manejo de la artrosis de cadera

\begin{tabular}{|c|c|c|c|c|}
\hline Manejo & $\begin{array}{l}\text { Fuertemente recomen- } \\
\text { dado }\end{array}$ & $\begin{array}{l}\text { Débil / condicionalmen- } \\
\text { te recomendado }\end{array}$ & Fuertemente NO recomendado & $\begin{array}{l}\text { Débil / condicionalmente NO } \\
\text { recomendado }\end{array}$ \\
\hline Farmacológico & $\begin{array}{l}\text { Antiinflamatorios no } \\
\text { esteroideos orales } \\
\text { (AINE), corticoides } \\
\text { intraarticulares }\end{array}$ & $\begin{array}{l}\text { Paracetamol, duloxeti- } \\
\text { na, tramadol }\end{array}$ & $\begin{array}{l}\text { Bifosfonatos, glucosamina, sulfato } \\
\text { de condroitina, hidroxicloroqui- } \\
\text { na, metotrexato, ácido hialurónico } \\
\text { intraarticular, plasma rico en pla- } \\
\text { quetas intraarticular, células madres } \\
\text { intraarticulares, biológicos }\end{array}$ & $\begin{array}{l}\text { Opioides distintos al tramadol, } \\
\text { colchicina, aceite de pescado, } \\
\text { vitamina D, toxina botulínica } \\
\text { intraarticular, proloterapia }\end{array}$ \\
\hline
\end{tabular}

Ton J, Perry D, Thomas B, et al. PEER umbrella systematic review of systematic reviews: Management of osteoarthritis in primary care. Can Fam Physician. 2020;66(3):e89-e98 .

Se realizó una revisión sistemática de revisiones sistemáticas (revisión panorámica u "overview") para determinar la eficacia y seguridad del manejo del dolor crónico secundario a artrosis en atención primaria ${ }^{4}$. Se realizó una búsqueda bibliográfica, hasta abril de 2019, en PubMed y Cochrane Library, utilizando como palabras claves "osteoarthritis" y "systematic review". Se incluyeron revisiones sistemáticas y meta-análisis de ensayos clínicos aleatorizados (ECAs) controlados con placebo que evaluaran al menos una de las siguientes intervenciones en adultos con artrosis más allá de vertebral: paracetamol, AINE orales o tópicos, inhibidores de la recaptación de serotonina y noradrenalina, antide- presivos tricíclicos, opioides, cannabinoides, glucosamina, condroitin, rubefacientes, inyecciones intraarticulares de plasma rico en plaquetas, viscosuplementación o corticoides, "counseling" y actividad física.

Se incluyeron 235 revisiones sistemáticas. La mejoría del dolor por intervención se presenta en la Tabla 2.

\section{Conclusiones del estudio}

La evidencia sugiere que la actividad física puede ser una intervención efectiva para el manejo del dolor en la artrosis (baja certeza de la evidencia), seguido en magnitud de efecto por la duloxetina y los AINE orales (moderada certeza de la evidencia). La eficacia de los corticoides intra-articulares es incierta y la respuesta del paracetamol fue similar al placebo. 
Tabla 2. Proporción de pacientes que logran una mejoría del dolor significativa (comparando tratamiento activo con control).

\begin{tabular}{|l|c|c|c|c|c|c|c|}
\hline Intervención & ECA & $\begin{array}{c}\text { Intervención: \% } \\
\text { mejoría significativa } \\
(\mathrm{n} / \mathrm{N})\end{array}$ & $\begin{array}{c}\text { Control: \% mejoría } \\
\text { significativa (n/N) }\end{array}$ & NNT & RR (IC 95\%) & $\begin{array}{c}\text { Seguimiento } \\
\text { (semanas) }\end{array}$ & $\begin{array}{c}\text { Certeza de la } \\
\text { evidencia } \\
\text { GRADE }\end{array}$ \\
\hline Actividad física & 11 & $47(341 / 723)$ & $21(138 / 644)$ & 4 & $2,36(1,79$ a 3,12) & 6 a 104 & Baja \\
\hline $\begin{array}{l}\text { Corticoides intraar- } \\
\text { ticulares }\end{array}$ & 7 & $50(203 / 410)$ & $31(91 / 296)$ & 6 & $1,74(1,15$ a 2,62$)$ & 4 a 24 & Muy Baja \\
\hline Duloxetina & 6 & $64(655 / 1.030)$ & $43(443 / 1.030)$ & 5 & $1,53(1,25$ a 1,87) & 10 a 18 & Moderada \\
\hline AINE (oral) & 43 & $57(12.201 / 21.495)$ & $39(2.838 / 7.294)$ & 6 & $1,44(1,36$ a 1,52$)$ & 4 a 104 & Moderada \\
\hline Glucosamina & 9 & $47(384 / 824)$ & $37(306 / 819)$ & 11 & $1,33(1,02$ a 1,74$)$ & 4 a 56 & Muy Baja \\
\hline AINE (tópico) & 22 & $61(2.357 / 3.892)$ & $47(1.602 / 3.373)$ & 8 & $1,27(1,16$ a 1,38$)$ & 1 a 12 & Baja \\
\hline Condroitin & 9 & $57(707 / 1.250)$ & $45(553 / 1.227)$ & 9 & $1,26(1,13$ a 1,41$)$ & 12 a 48 & Moderada \\
\hline Viscosuplementación & 31 & $53(1.748 / 3.291)$ & $44(1.300 / 2.963)$ & 11 & $1,22(1,12$ a 1,33$)$ & 2 a 160 & Muy baja \\
\hline Opioides (oral) & 15 & $47(1.795 / 3.854)$ & $43(1.048 / 2.412)$ & 32 & $1,16(1,02$ a 1,32$)$ & 10 a 24 & Muy baja \\
\hline Paracetamol & 2 & $47(240 / 513)$ & $43(204 / 478)$ & - & $1,17(0,83$ a 1,64$)$ & 6 a 24 & Baja \\
\hline
\end{tabular}

ECA: ensayos clínicos aleatorizados; IC: intervalo de confianza; NNT: número necesario a tratar; RR: riesgo relativo.

Goh SL, Persson MSM, Stocks J, et al. Relative Efficacy of Different Exercises for Pain, Function, Performance and Quality of Life in Knee and Hip Osteoarthritis: Systematic Review and Network Meta-Analysis. Sports Med. 2019;49(5):743-761

Este meta-análisis en red Bayesiano ${ }^{5}$ investigó la eficacia relativa de diferentes tipos de actividad física (aeróbica, ejercicios de mente-cuerpo como yoga o Tai Chi, fortalecimiento, flexibilidad, mixtas) en el tratamiento del dolor, la función, el desempeño y la calidad de vida de pacientes con artrosis de rodilla y de cadera. Se realizó una búsqueda hasta diciembre de 2017 en nueve fuentes (AMED, CENTRAL, CINAHL, EMBASE, MEDLINE, PEDro, PubMed, SPORTDiscus y Google Scholar) de ECA que evaluaran el efecto de la actividad física, sin otro tratamiento asociado, en cuanto a dolor, funcionalidad, desempeño objetivo o calidad de vida en adultos con artrosis de rodilla o cadera diagnosticada clínica o radiológicamente.

Se incluyeron 103 ECA (9.134 participantes), y se observó un importante efecto en todos los casos, expresado en unidades de error estándar con su intervalo de credibilidad del $95 \%$, siendo la actividad aeróbica la más efectiva para el manejo del dolor $(1,11$; $0,69$ a 1,54$)$ y el desempeño $(1,05 ; 0,63$ a 1,48$)$. El efecto de la actividad física la calidad de vida fue menos marcado. Los ejercicios de mente-cuerpo tuvieron una respuesta similar en el manejo del dolor $(1,11 ; 0,63$ a 1,59), con mejor respuesta en la funcionalidad $(0,81 ; 0,27$ a 1,36$)$. En cuanto a los ejercicios de fortaleci- miento y flexibilidad tuvieron también buena respuesta en varios aspectos pero en un nivel moderado. La actividad física mixta fue la menos efectiva en todos los resultados evaluados.

\section{Conclusiones del estudio}

Para manejo del dolor, función y desempeño (y no tanto para la calidad de vida), todos los tipos de actividad física fueron significativamente mejores que los cuidados habituales, siendo mayor el efecto en los ejercicios aeróbicos y de mente-cuerpo, seguidos por los ejercicios de fortalecimiento o estiramiento.

\section{Conclusiones y recomendaciones}

Existen numerosas estrategias útiles para el manejo del dolor en pacientes con artrosis de cadera.

En el caso de la paciente, una opción válida inicial sería la indicación de AINE por un período corto e inicio de actividad física. Sería necesario plantear junto con la paciente que tipo de ejercicio es más compatible con sus gustos y capacidades para asegurar mayor adherencia, siendo los más recomendados la actividad aeróbica y el Tai Chi o yoga. En caso de persistencia del dolor se podría escalar a tramadol o duloxetina, siempre asociado con la actividad física.

Recibido el 05/12/2020 Aceptado el 20/02/202 y Publicado el 04/06/2021.

\section{Referencias}

1. Lespasio M, Sultan AA, Piuzzi NS, et al. Hip Osteoarthritis: A Primer. Perm J. 2018;22:17-084. Available from: 10.7812/tpp/17-084;https://dx.doi. org/10.7812/tpp/17-084.

2. Jordan JM, Helmick CG, Renner JB. Prevalence of hip symptoms and radiographic and symptomatic hip osteoarthritis in African Americans and Caucasians: the Johnston County Osteoarthritis Project. J Rheumatol. 2009;36(4):809-815. Available from: 10.3899/jrheum.080677.

3. Kolasinski SL, Neogi T, Hochberg MC, et al. 2019 American College of Rheumatology/Arthritis Foundation Guideline for the Management of Osteoarthritis of the Hand, Hip, and Knee. Arthritis Care Res (Hoboken). 2020;72(2):149-162. Available from: 10.1002/acr.24131.

4. Ton J, Perry D, Thomas B, et al. PEER umbrella systematic review of systematic reviews: Management of osteoarthritis in primary care. Can Fam Physician. 2020;66(3):89-98.

5. Goh SL, Persson MSM, Stocks J, et al. Relative Efficacy of Different Exercises for Pain, Function, Performance and Quality of Life in Knee and Hip Osteoarthritis: Systematic Review and Network Meta-Analysis. Sports Med. 2019;49(5):743-761. Available from: 10.1007/s40279-019-01082-0; https://dx.doi.org/10.1007/s40279-019-01082-0. 